\title{
Food components affecting the oil absorption and crispness of fried batter
}

\begin{abstract}
Frying batters are used to add value to a product by improving texture, flavour, weight and volume and reducing water loss during frying which, in turn, lessens oil absorption. The physical characteristics of fried batters were studied using model systems based on rice flours. Crispness was positively correlated with amylose content, while oil absorption was negatively correlated with amylose content. Addition of pregelatinised rice flour improved crispness but resulted in increased oil absorption because of the porous nature of the fried product. Amongst the proteins (egg yolk, gluten, skimmed milk, whey and ovalbumin) studied, ovalbumin was able to reduce oil absorption and improve the crispness of the fried batter. Addition of calcium chloride also reduced oil absorption and improved crispness, while a little oil reduced the oil content of the fried batter and improved its taste. Addition of ovalbumin, oil or calcium chloride beyond the optimal levels reduced crispness and increased oil absorption in a linear manner. Modified tapioca starch and diglyceride emulsifiers improved crispness but increased oil absorption. The amount of water added to the formulation affected the physical characteristics of the fried batter. The optimal formulation for a crisp frying batter with reasonably low oil absorption was an amylose/amylopectin ratio of about 18 : 67; with (g kg-1) 850 pregelatinised rice flour; up to 150 modified tapioca starch; 30 ovalbumin; 1 calcium chloride; 20 oil; 20 emulsifier and a water/flour ratio of 2:1.
\end{abstract}

Keyword: Rice; Protein; Starch; Calcium; Batter; Oil uptake; Crispness 